\title{
Supplementary 3: Bayesian estimation of the posterior probability of game players' in-game behaviors
}

Quan-Hoang Vuong ${ }^{1,2, *}$, Manh-Toan Ho ${ }^{1,3}$, Minh-Hoang Nguyen ${ }^{1,3}$, Thanh-Hang Pham ${ }^{4,5}$, HoangAnh $\mathrm{Ho}^{1}$, Thu-Trang Vuong ${ }^{6}$, Viet-Phuong La ${ }^{1,3}$

1 Centre for Interdisciplinary Social Research, Phenikaa University, Yen Nghia Ward, Ha Dong District, Hanoi 100803, Vietnam

${ }^{2}$ Centre Emile Bernheim, Université Libre de Bruxelles, 1050 Brussels, Belgium

${ }^{3}$ A.I. for Social Data Lab, Vuong \& Associates, 3/161 Thinh Quang, Dong Da District, Hanoi, 100000, Viet Nam

${ }^{4}$ Faculty of Management and Tourism, Hanoi University, Km9, Nguyen Trai Road, Thanh Xuan, Hanoi 100803, Vietnam

${ }^{5}$ School of Business, RMIT Vietnam University, Hanoi, 100000, Viet Nam

${ }^{6}$ Sciences Po Paris, 27 Rue Saint-Guillaume, 75007 Paris, France

*Contact: Quan-Hoang Vuong (qvuong@ulb.ac.be); Manh-Toan Ho (toan.homanh@phenikaauni.edu.vn)

\section{Aim}

In this Supplementary file, we present the materials, mathematical formulas, codes, results, and interpretation of the estimated posterior probability of game players' in-game environmental behaviors. We also compare these behaviors of players with differences in terms of geographical region, biological sex, and the highest educational level completed. To see the preparation of analyses' materials, please refer to Supplementary 1.

\section{Materials}

To estimate the probability of players' in-game environmental behaviors, we employed three different types of demographic variables (geographical region, biological sex, and educational level) and the two variables of game players' behaviors towards the environment (animal and plant collection) using two different datasets (the dataset [1] with 584 responses and [2] with 503 responses, respectively).

Variable "A1” - region contains four levels: 'Asia', 'EU', 'US/Canada', and 'Other'.

Variable "A2" - sex contains two levels: 'Male' and 'Female'.

Variable "A3" - education contains four levels: 'Secondary school', 'High school', 'Undergraduate school', and 'Graduate school and higher'.

The "Animal" variable was created by combining "E6" and "E7" variables, while "Tree" variable was created by combining "E8" and "E9" variables. "E6", "E7", "E8", and "E9" were variables representing the likelihood to collect the following species: Fish, bug, tree, and flower, respectively. These four variables were created from the questions "Do you want to collect or plant all the following species in the game?" with a 5-point Likert scale ranging from 'Very unlikely' to 
'Very likely'. However, in this analysis, the 'Maybe' level was excluded and the other four levels were combined to obtain binary variables with two levels: 'Unlikely' and 'Likely'. The dataset [1] and [2] after discarding 'Maybe' level consisted of 377 responses and 317 responses, respectively.

\section{Model construction and results}

\subsection{Posterior probability of in-game environmental behaviors}

\subsubsection{Behavior towards animals}

The formula for estimating the posterior probability of in-game behaviors towards animals are as follows:

$$
\text { Animal bernoulli(theta_Animal) }
$$

The formula mentioned above can be coded and simulated with the prior set as normal distribution at 0.3 using the following commands of the bayesvl R package:

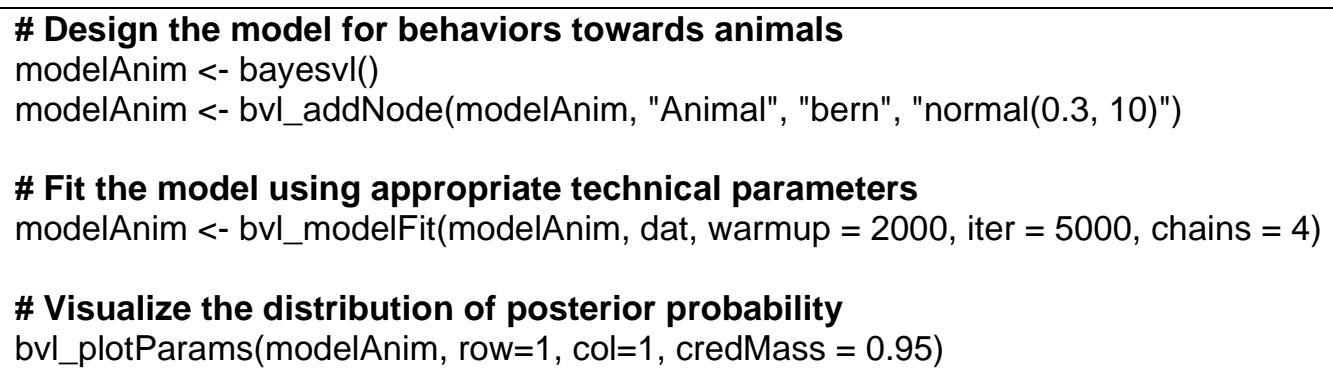

In order to ensure the robustness, tweaking the priors of each model and performing posterior estimation with two different datasets are employed. First of all, using dataset [1], we estimate the posterior probability under two different prior set-ups with mean at 0.3 and 0.6 , which are equivalent to the 'unlikely' and 'likely' to collect all animals, respectively. Then, we replicate the posterior estimation process with the dataset [2] for the second sensitivity check.

Table S3_1: Posterior probability of the in-game behavior towards animal with the prior being Normal $(0.3,10)$ and Normal $(0.6,10)$

\begin{tabular}{|c|c|c|c|c|c|c|c|c|c|c|}
\hline \multicolumn{11}{|c|}{ Prior: theta Animal normal $(0.3,10)$} \\
\hline Dataset & Parameter & mean & sd & $2.5 \%$ & $25 \%$ & $50 \%$ & $75 \%$ & $97.5 \%$ & n_eff & Rhat \\
\hline dataset [1] & theta_animal & 0.97 & 0.01 & 0.95 & 0.97 & 0.97 & 0.98 & 0.99 & 4354 & 1 \\
\hline dataset [2] & theta_animal & 0.97 & 0.01 & 0.95 & 0.96 & 0.97 & 0.98 & 0.98 & 4624 & 1 \\
\hline \multicolumn{11}{|c|}{ Prior: theta Animal normal $(0.6,10)$} \\
\hline Dataset & Parameter & mean & sd & $2.5 \%$ & $25 \%$ & $50 \%$ & $75 \%$ & $97.5 \%$ & n_eff & Rhat \\
\hline dataset [1] & theta_animal & 0.97 & 0.01 & 0.95 & 0.97 & 0.97 & 0.98 & 0.99 & 4342 & 1 \\
\hline dataset [2] & theta_animal & 0.97 & 0.01 & 0.95 & 0.96 & 0.97 & 0.98 & 0.98 & 4612 & 1 \\
\hline
\end{tabular}

Table S3_1 shows the posterior estimation results of the posterior probability of the likelihood to collect all animals under two different prior set-ups employing datasets [1] and [2]. In all circumstances, the values of sample size (n_eff) were greater than 1000, hinting at well-mixing chains (McElreath, 2020). Meanwhile, the Gelman Shrink Factor values of all parameters (Rhat) were equal to 1 , indicating good signals of convergence (Brooks \& Gelman, 1998). The probability distribution generated from two different datasets are illustrated in Figure S3_1. 


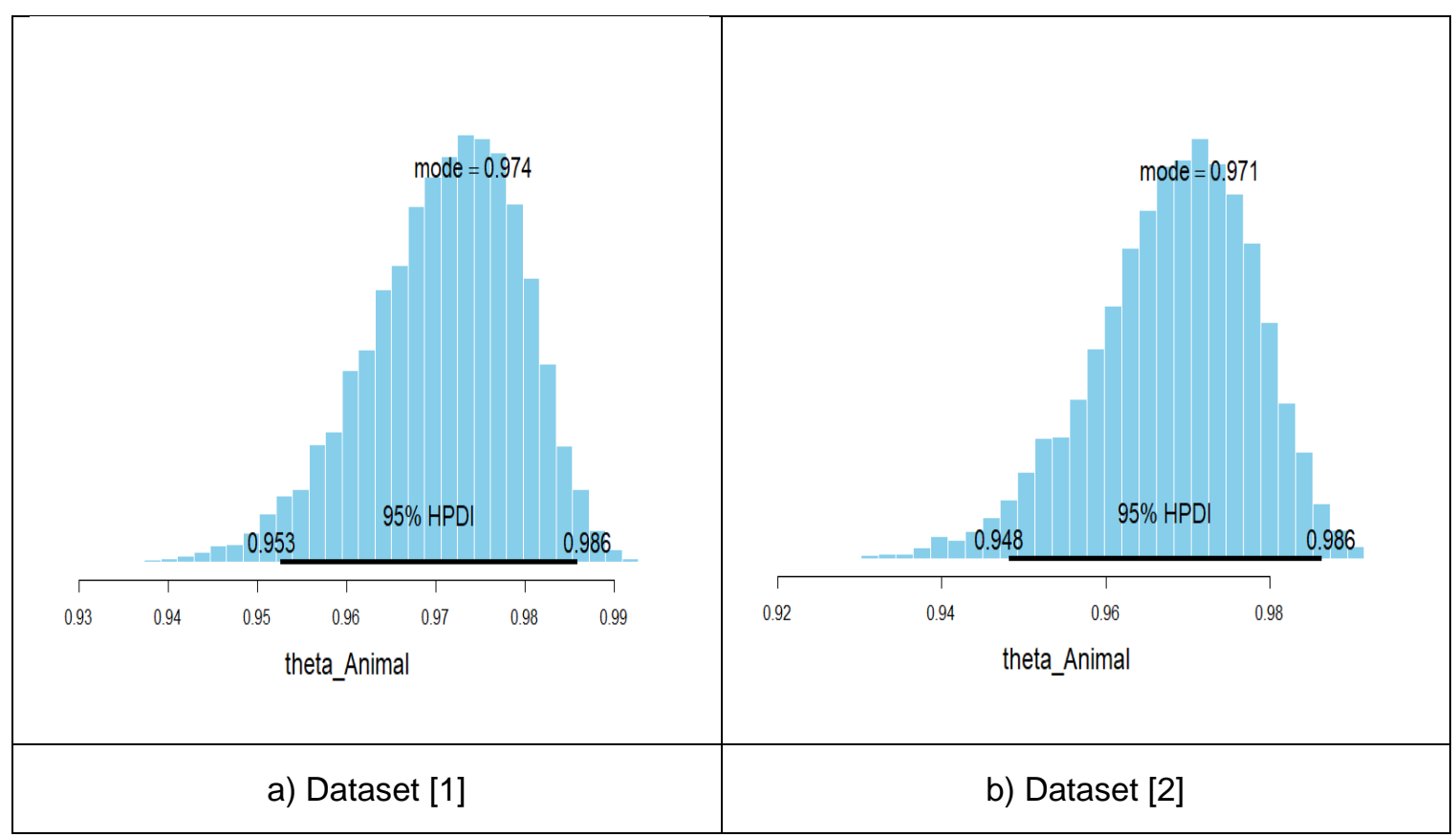

Figure S3_1: The distribution of the posterior probability of the in-game behavior towards animals with the prior being Normal $(0.3,10)$.

Figure S3_1a) and b) represent the probability distribution generated from the dataset [1] and [2], respectively. The posterior probabilities of both cases are normally distributed at around 0.97 , suggesting the simulation's robustness despite the change in sample size.

\subsubsection{Behavior towards plants}

The formula for estimating the posterior probability of in-game behaviors towards plants are as follows:

$$
\text { Tree } \sim \text { bernoulli(theta_Tree) }
$$

The formula mentioned above can be coded and simulated with the prior set as normal distribution at 0.3 using the following commands of the bayesvl R package (La \& Vuong, 2019; Q.H. Vuong, La, Nguyen, Ho, Ho, et al., 2020; Q.-H. Vuong, La, Nguyen, Ho, Tran, et al., 2020):

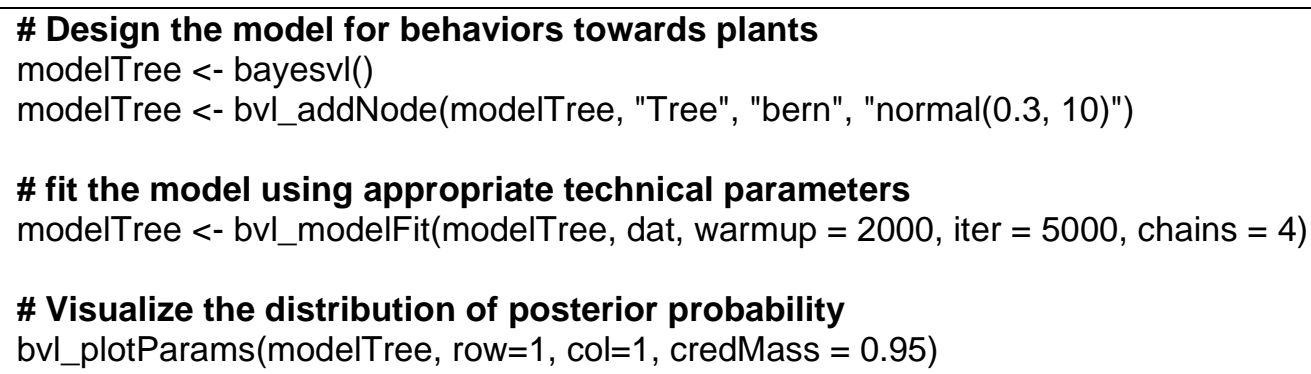

Similar to the above sub-section, tweaking the priors of each model and performing posterior estimation with two different datasets are employed in order to ensure the robustness. First of all, using the dataset [1], we estimate the posterior probability under two different prior setups with mean at 0.3 and 0.6 , which are equivalent to the 'unlikely' and 'likely' to collect all plants, 
respectively. Then, we replicate the posterior estimation process with the dataset [2] for the second sensitivity check. The reporting of the results and limitations strictly follows the principle of $\mathrm{Q}$. $\mathrm{H}$. Vuong (2020).

Table S3_2: Posterior probability of the in-game behavior towards plants with the prior being Normal $(0.3,10)$ and Normal $(0.6,10)$

\begin{tabular}{|c|c|c|c|c|c|c|c|c|c|c|}
\hline \multicolumn{11}{|c|}{ Prior: theta Tree $\sim$ normal $(0.3,10)$} \\
\hline Dataset & Parameter & mean & sd & $2.5 \%$ & $25 \%$ & $50 \%$ & $75 \%$ & $97.5 \%$ & n_eff & Rhat \\
\hline dataset [1] & theta_Tree & 0.96 & 0.01 & 0.94 & 0.95 & 0.96 & 0.97 & 0.98 & 4541 & 1 \\
\hline dataset [2] & theta_Tree & 0.96 & 0.01 & 0.93 & 0.95 & 0.96 & 0.96 & 0.98 & 4686 & 1 \\
\hline \multicolumn{11}{|c|}{ Prior: theta_Tree $\sim \operatorname{normal}(0.6,10)$} \\
\hline Dataset & Parameter & mean & sd & $2.5 \%$ & $25 \%$ & $50 \%$ & $75 \%$ & $97.5 \%$ & n_eff & Rhat \\
\hline dataset [1] & theta_Tree & 0.96 & 0.01 & 0.94 & 0.95 & 0.96 & 0.97 & 0.98 & 3719 & 1 \\
\hline dataset [2] & theta_Tree & 0.96 & 0.01 & 0.93 & 0.95 & 0.96 & 0.96 & 0.98 & 4409 & 1 \\
\hline
\end{tabular}

Table S3_2 shows the posterior estimation results of the posterior probability of the likelihood to collect all plants under two different prior set-ups employing datasets [1] and [2]. In all circumstances, the values of sample size (n_eff) were greater than 1000, hinting at well-mixing chains. Meanwhile, the Gelman Shrink Factor values of all parameters (Rhat) were equal to 1 , indicating good signals of convergence. The probability distribution generated from two different datasets are illustrated in Figure S3_2.

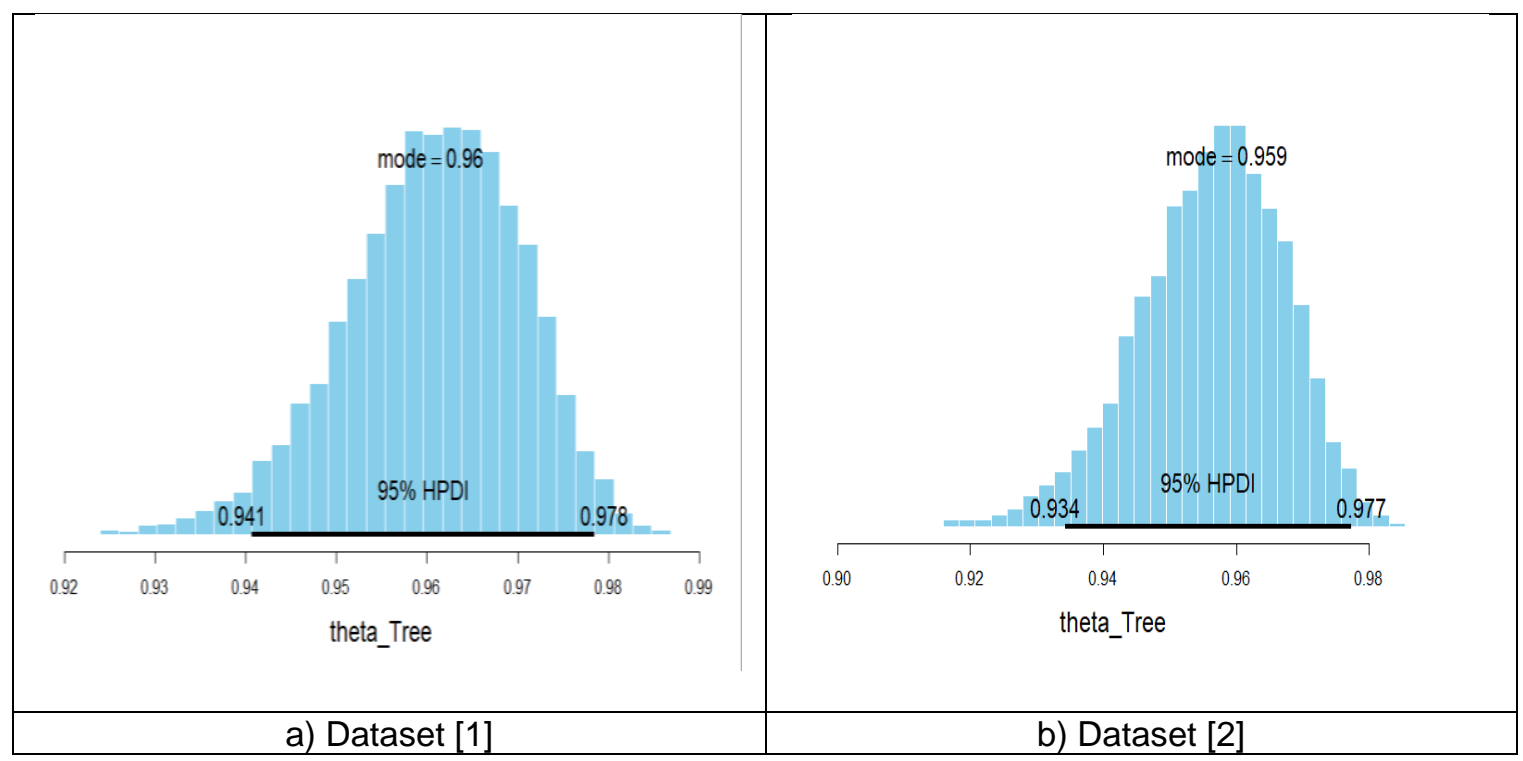

Figure S3_2: The distribution of the posterior probability of the in-game behavior towards plants with the prior being Normal $(0.3,10)$.

Figure S3_2a) and b) represent the distribution generated from the dataset [1] and [2], respectively. The posterior probabilities of both cases are normally distributed at around 0.96 , suggesting the simulation's robustness despite the change in sample size.

The comparison between the densities of the posterior probability of collecting all animals and plants is illustrated in Figure S3_3. 


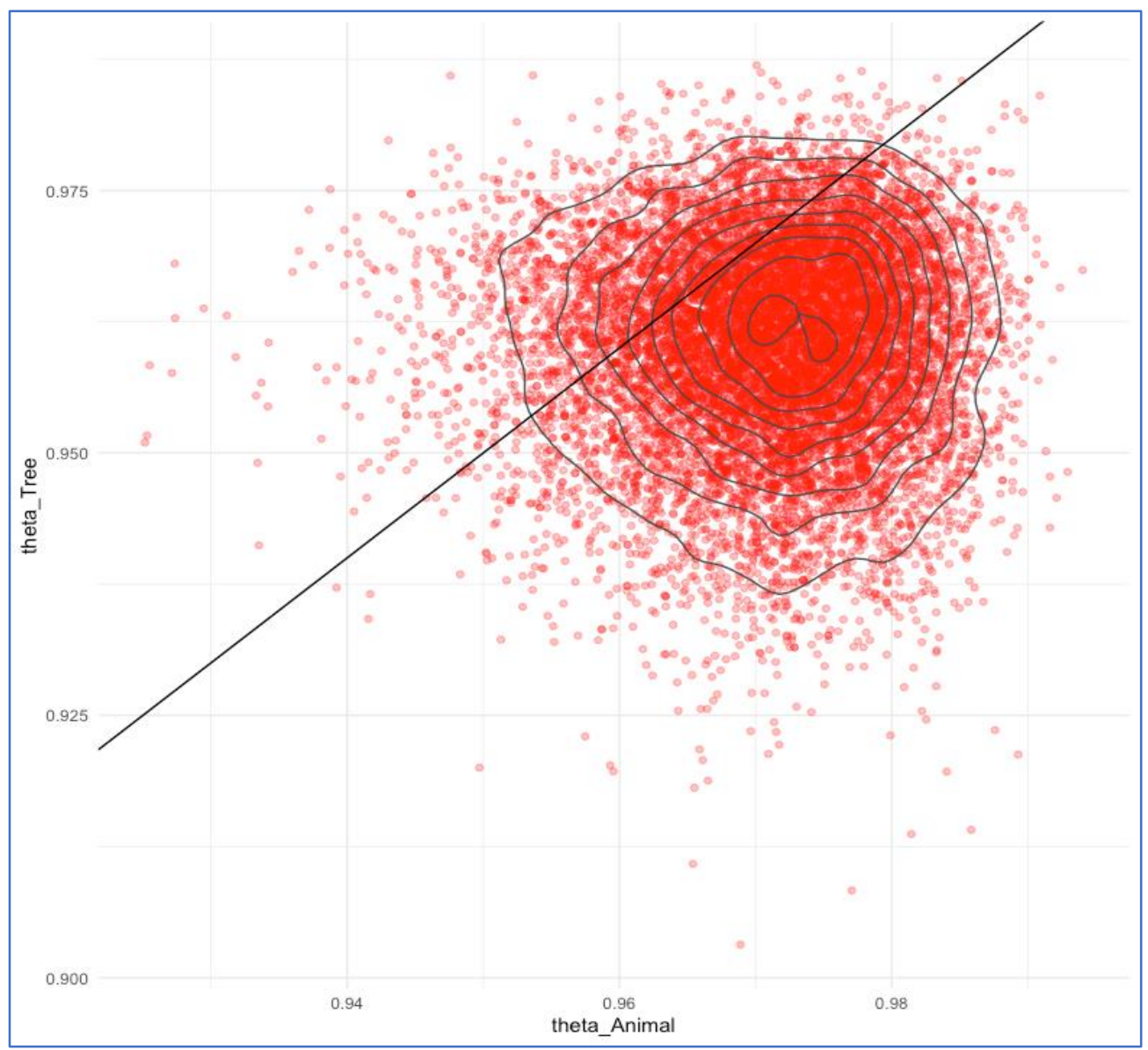

Figure S3_3: The comparative densities between "theta_Animal" and "theta_Tree" estimated with the prior being Normal $(0.3,10)$.

Figure S3_3 shows relatively similar probabilistic trends of in-game behaviors towards animals and plants, with game players more likely to collect all animals than plants. Although a slight difference exists between two behaviors, it is negligible.

\subsection{Posterior probability of in-game environmental behaviors by geographical regions}

The formulas for estimating the posterior probability of in-game behaviors towards animals and plants by geographical regions are as follows:

$$
\begin{gathered}
\text { Anim } \sim \text { bernoulli(theta_Anim[Region]); } \\
\text { Tree } \sim \text { bernoulli(theta_Tree[Region]) }
\end{gathered}
$$

Where:

- theta[1] Asia

- theta[2] EU

- theta[3] Other

- theta[4] US/Canada 
Two formulas mentioned above can be coded and simulated using the following commands of the rstan R package (Stan Development Team, 2020):

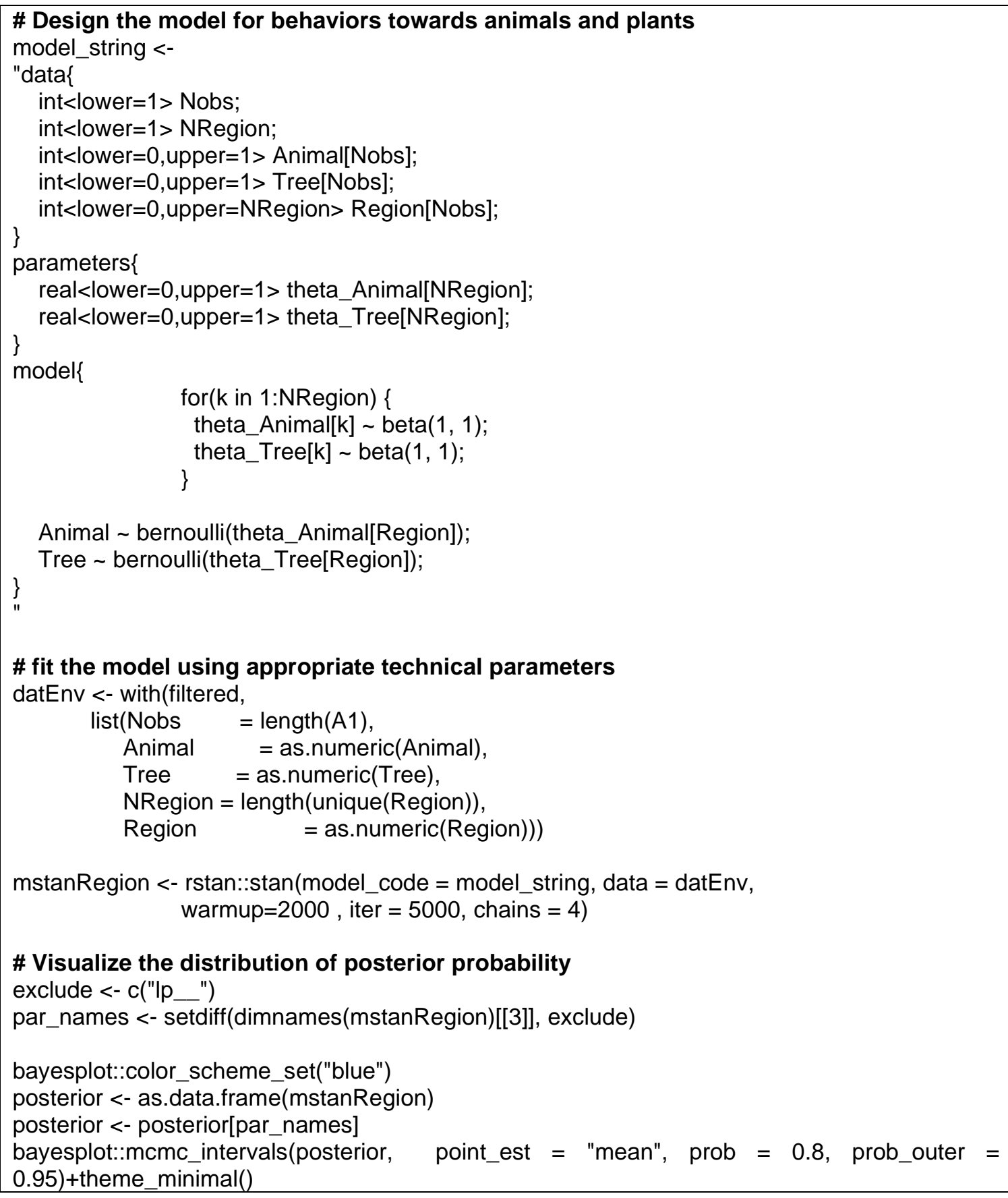

Animal bernoulli(theta_Animal[Region]);

Tree $\sim$ bernoulli(theta_Tree[Region]);

\# fit the model using appropriate technical parameters

Table S3_3 presents the posterior probability of collecting all animals and plants of respondents from four different regions: Asia, EU, US/Canada, and Others employing two different datasets. The n_eff and Rhat indicate the good convergence of posteriors. The probability distribution of each region generated from datasets [1] and [2] is shown in Figure S3_4. 
Table S2_3: Posterior probability of the in-game behavior towards animals and plants by geographical regions with the prior being Beta $(1,1)$.

\begin{tabular}{|c|c|c|c|c|c|c|c|c|c|c|}
\hline Dataset & Parameter & mean & sd & $2.5 \%$ & $25 \%$ & $50 \%$ & $75 \%$ & $97.5 \%$ & n_eff & Rhat \\
\hline \multirow{8}{*}{ Dataset [1] } & Theta_Anim [1] & 0.95 & 0.02 & 0.90 & 0.93 & 0.95 & 0.96 & 0.98 & 21136 & 1 \\
\hline & Theta_Anim [2] & 0.96 & 0.02 & 0.90 & 0.94 & 0.96 & 0.97 & 0.99 & 18663 & 1 \\
\hline & Theta_Anim [3] & 0.94 & 0.06 & 0.78 & 0.91 & 0.95 & 0.98 & 1.00 & 16989 & 1 \\
\hline & Theta_Anim [4] & 0.98 & 0.01 & 0.95 & 0.97 & 0.98 & 0.99 & 0.99 & 19288 & 1 \\
\hline & Theta_Tree [1] & 0.95 & 0.02 & 0.90 & 0.93 & 0.95 & 0.96 & 0.98 & 19791 & 1 \\
\hline & Theta_Tree [2] & 0.97 & 0.02 & 0.92 & 0.96 & 0.98 & 0.99 & 1.00 & 20479 & 1 \\
\hline & Theta_Tree [3] & 0.88 & 0.08 & 0.69 & 0.83 & 0.89 & 0.94 & 0.98 & 18359 & 1 \\
\hline & Theta_Tree [4] & 0.96 & 0.01 & 0.92 & 0.95 & 0.96 & 0.97 & 0.98 & 19807 & 1 \\
\hline \multirow{8}{*}{ Dataset [2] } & Theta_Anim [1] & 0.94 & 0.03 & 0.87 & 0.92 & 0.94 & 0.96 & 0.98 & 20151 & 1 \\
\hline & Theta_Anim [2] & 0.96 & 0.03 & 0.89 & 0.94 & 0.96 & 0.97 & 0.99 & 18777 & 1 \\
\hline & Theta_Anim [3] & 0.92 & 0.07 & 0.74 & 0.89 & 0.95 & 0.98 & 1.00 & 17864 & 1 \\
\hline & Theta_Anim [4] & 0.98 & 0.01 & 0.95 & 0.97 & 0.98 & 0.99 & 0.99 & 18624 & 1 \\
\hline & Theta_Tree [1] & 0.94 & 0.03 & 0.88 & 0.92 & 0.94 & 0.96 & 1.00 & 19571 & 1 \\
\hline & Theta_Tree [2] & 0.96 & 0.02 & 0.92 & 0.96 & 0.97 & 0.99 & 1.00 & 18038 & 1 \\
\hline & Theta_Tree [3] & 0.85 & 0.10 & 0.62 & 0.79 & 0.87 & 0.92 & 0.98 & 18341 & 1 \\
\hline & Theta_Tree [4] & 0.95 & 0.02 & 0.92 & 0.94 & 0.95 & 0.96 & 0.98 & 18390 & 1 \\
\hline
\end{tabular}

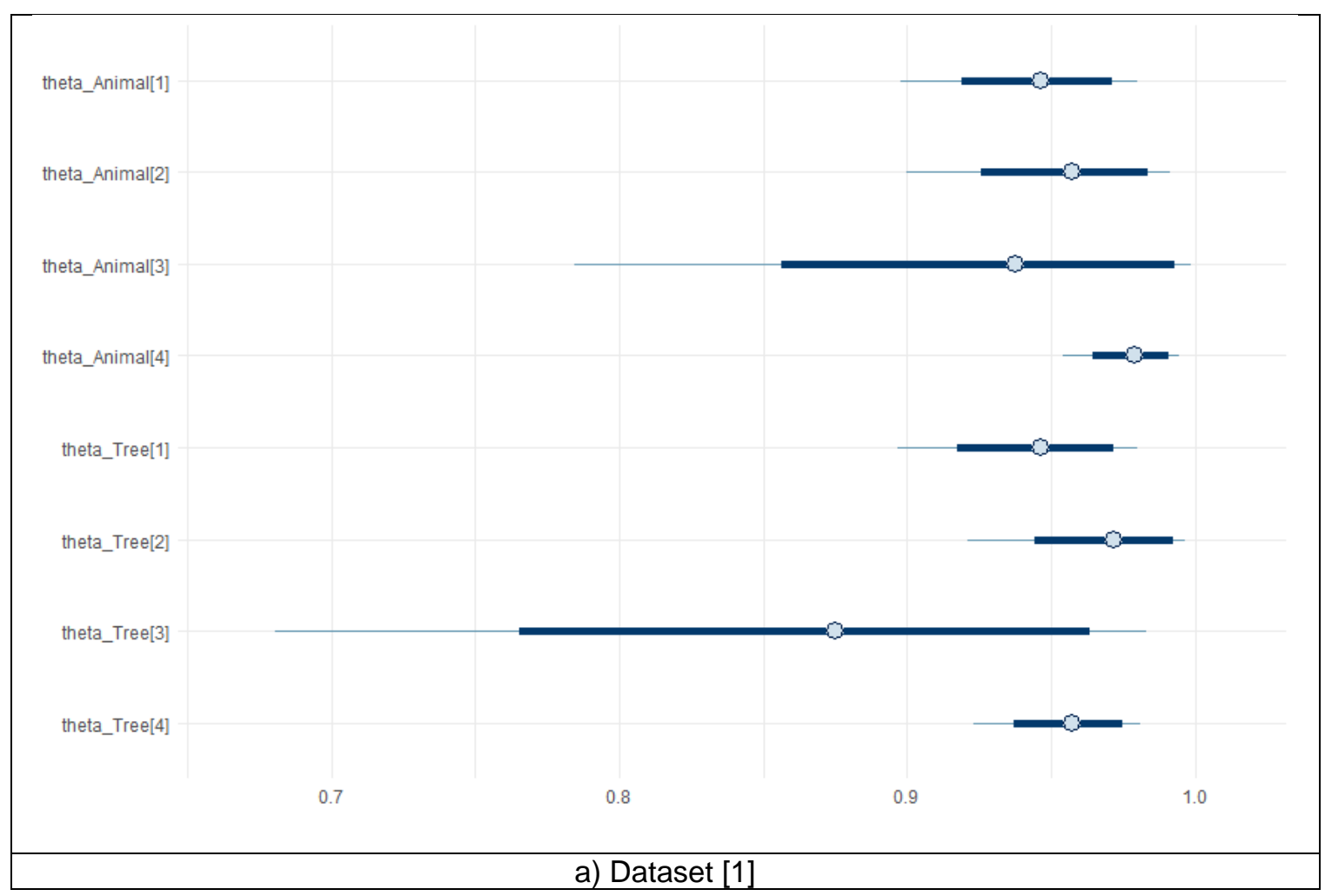




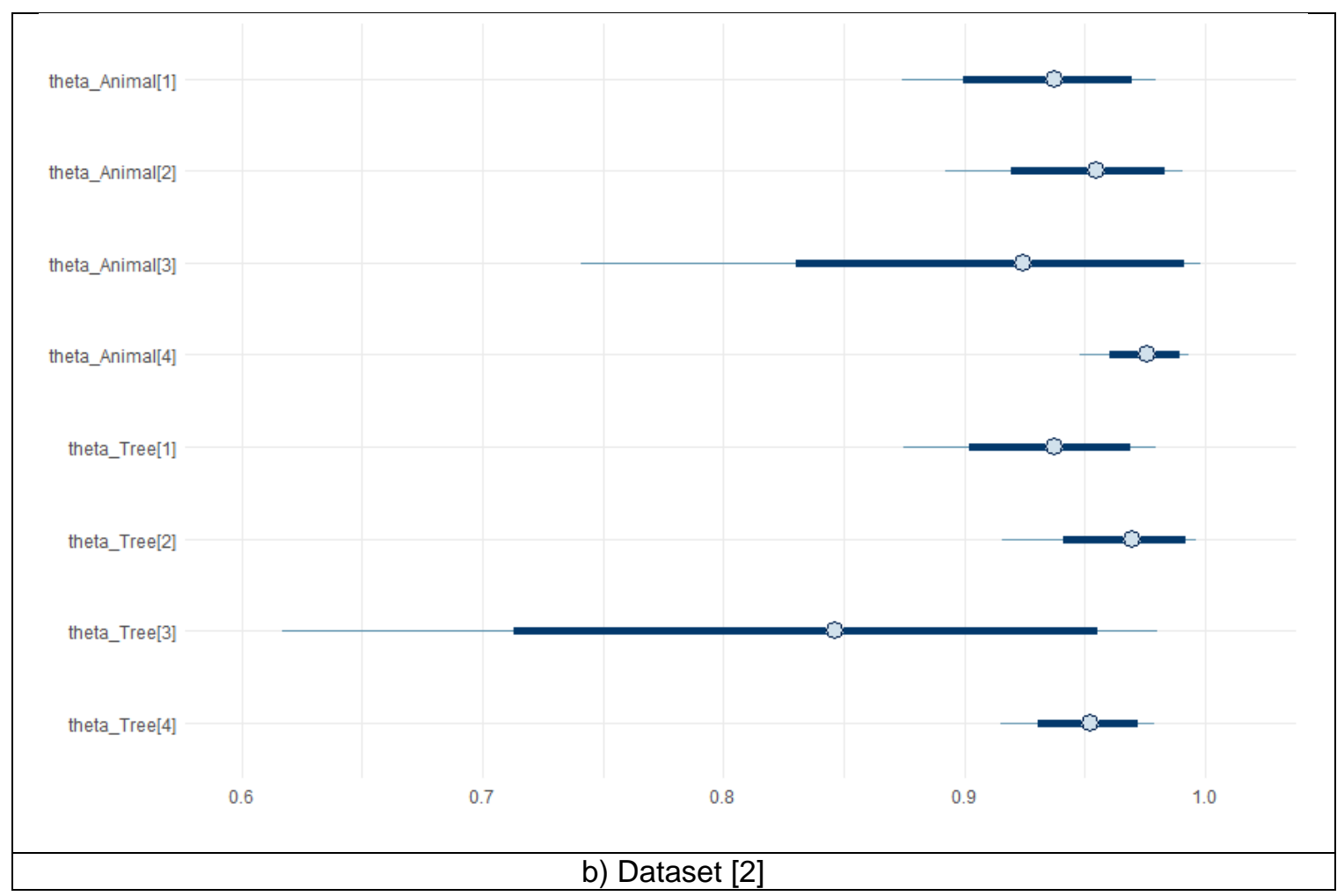

Figure S3_4: The distribution of the posterior probability of the in-game behavior towards animals and plants by regions with the prior being Beta $(1,1)$.

Figure S3_4a) and b) represent the distribution generated from the dataset [1] and [2], respectively. There exists the disparity of posterior probabilities among regions, with respondents from the US/Canada obtaining the highest likelihood, but the degree of difference is negligible. The sensitivity when applying the model on the different datasets is also negligible.

\subsection{Posterior probability of in-game environmental behaviors across biological sexes}

The formulas for estimating the posterior probability of in-game behaviors towards animals and plants across biological sexes are as follows:

$$
\begin{aligned}
& \text { Anim } \sim \text { bernoulli(theta_anim[Sex]); } \\
& \text { Tree } \sim \text { bernoulli(theta_tree[Sex]); }
\end{aligned}
$$

Where:

- theta[1] Male

- theta[2] Female

Two formulas mentioned above can be coded and simulated using the following commands of the rstan R package (Stan Development Team, 2020):

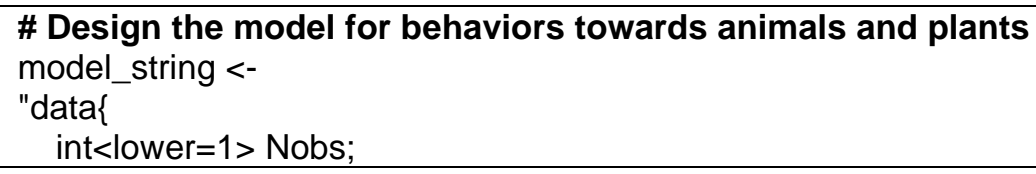




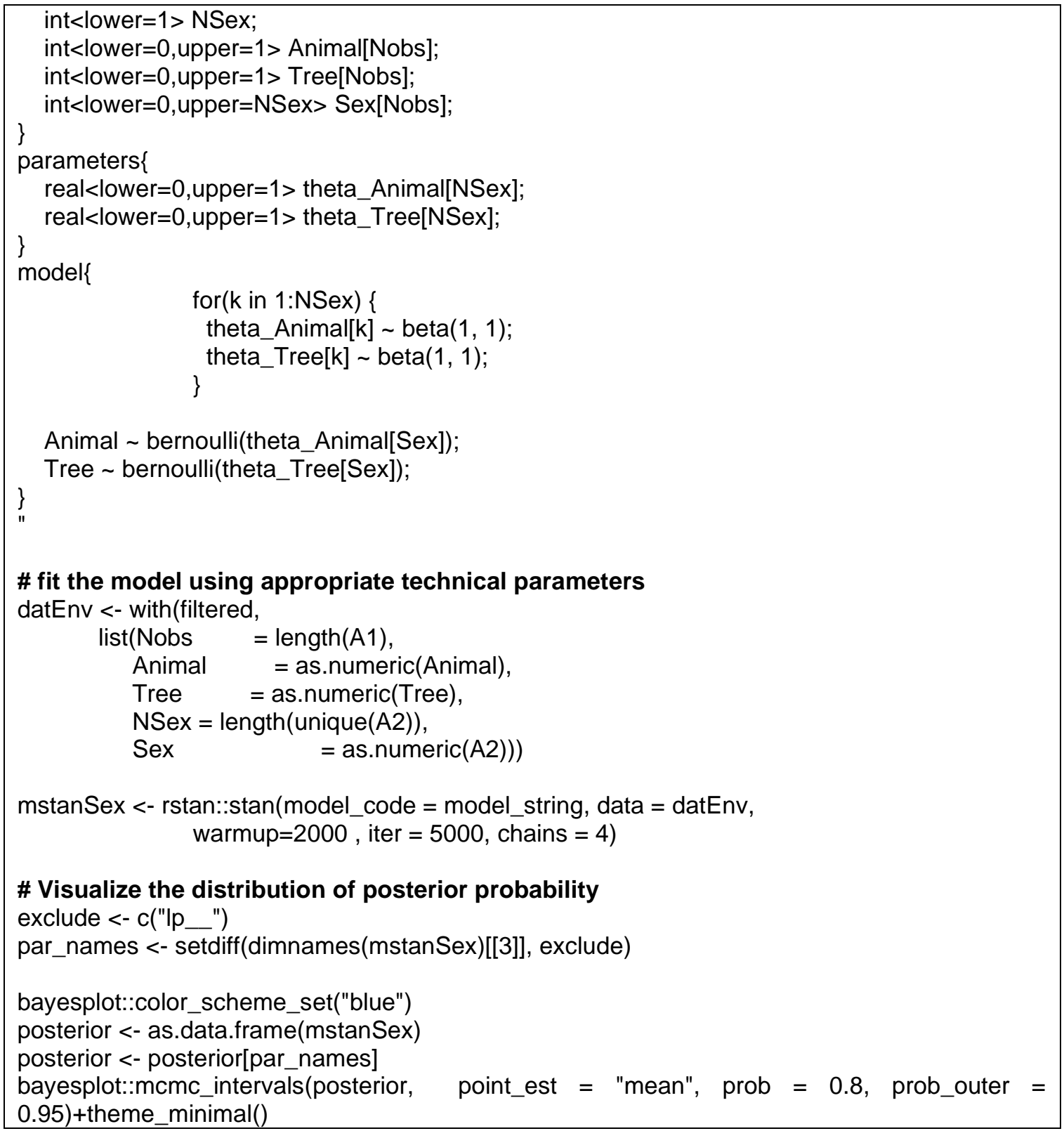

Table S3_4 presents the posterior probability of collecting all animals and plants by biological sex, employing two different datasets. The n_eff and Rhat indicate the good convergence of posteriors. The probability distribution of each sex generated from datasets [1] and [2] is shown in Figure S3_5.

Table S3_4: Posterior probability of the in-game behavior towards animals and plants by biological sex with the prior being Beta $(1,1)$.

\begin{tabular}{|c|c|c|c|c|c|c|c|c|c|c|}
\hline Dataset & Parameter & mean & sd & $\mathbf{2 . 5 \%}$ & $\mathbf{2 5 \%}$ & $\mathbf{5 0} \%$ & $\mathbf{7 5} \%$ & $\mathbf{9 7 . 5} \%$ & $\mathbf{n}$ eff & Rhat \\
\hline \multirow{3}{*}{ Dataset [1] } & Theta_Anim [1] & 0.95 & 0.02 & 0.89 & 0.93 & 0.95 & 0.96 & 0.98 & 13744 & 1 \\
\cline { 2 - 10 } & Theta_Anim [2] & 0.98 & 0.01 & 0.96 & 0.97 & 0.98 & 0.98 & 0.99 & 13853 & 1 \\
\cline { 2 - 10 } & Theta_Tree [1] & 0.94 & 0.03 & 0.88 & 0.92 & 0.94 & 0.96 & 0.98 & 13586 & 1 \\
\hline
\end{tabular}




\begin{tabular}{|l|l|l|l|l|l|l|l|l|l|l|}
\hline & Theta_Tree [2] & 0.97 & 0.01 & 0.94 & 0.96 & 0.97 & 0.97 & 0.98 & 14293 & 1 \\
\hline & Theta_Anim [1] & 0.94 & 0.03 & 0.88 & 0.93 & 0.94 & 0.96 & 0.98 & 13844 & 1 \\
\cline { 2 - 10 } Dataset [2] & Theta_Anim [2] & 0.98 & 0.01 & 0.95 & 0.97 & 0.98 & 0.98 & 0.99 & 13787 & 1 \\
\cline { 2 - 10 } & Theta_Tree [1] & 0.93 & 0.03 & 0.86 & 0.91 & 0.93 & 0.95 & 0.97 & 13085 & 1 \\
\cline { 2 - 10 } & Theta_Tree [2] & 0.96 & 0.01 & 0.94 & 0.96 & 0.96 & 0.97 & 0.98 & 12893 & 1 \\
\hline
\end{tabular}

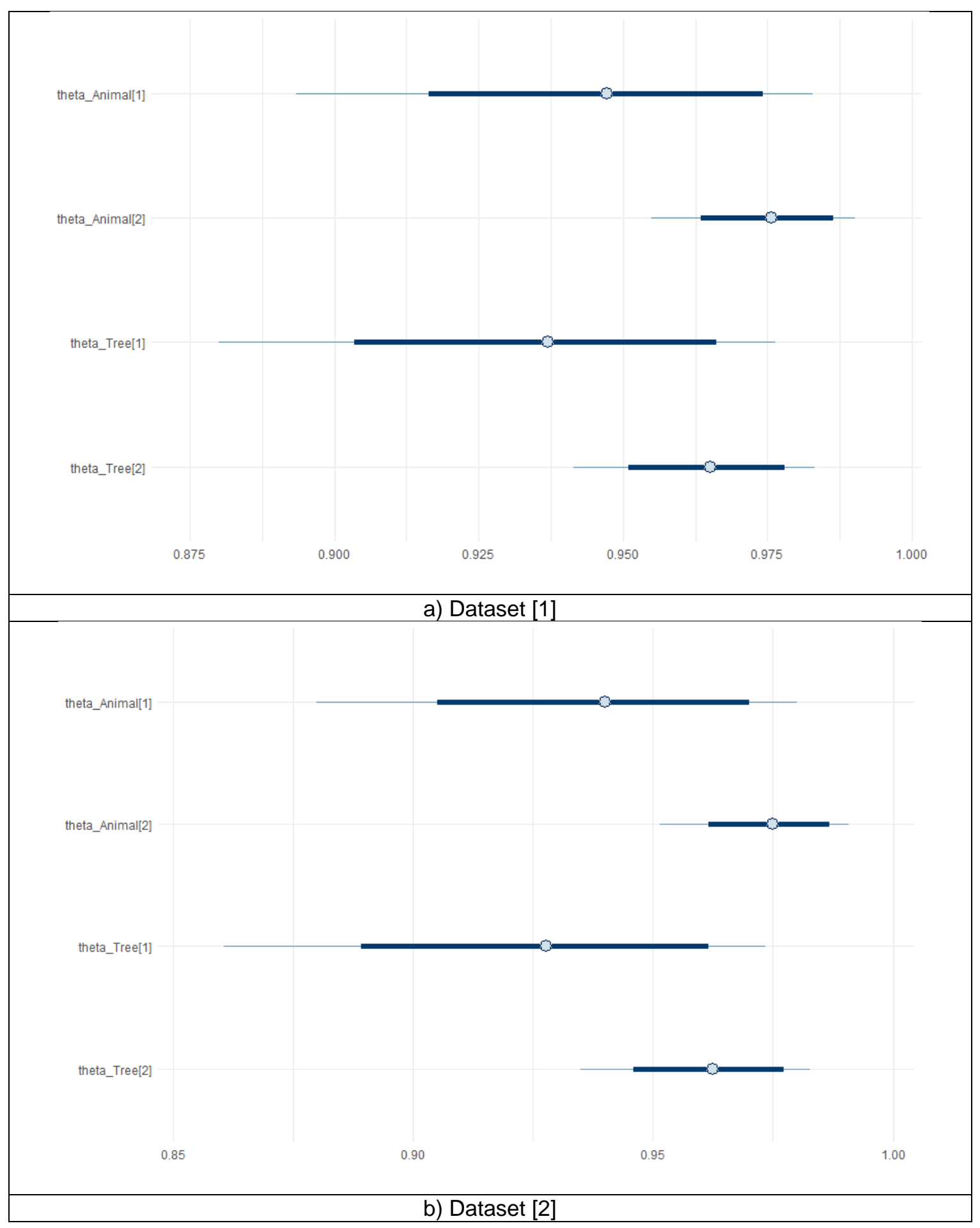


Figure S3_5: The distribution of the posterior probability of the in-game behavior towards animals and plants by sex with the prior being Beta $(1,1)$.

Figure S3_5a) and b) represent the distribution generated from the dataset [1] and [2], respectively. There exists the disparity of posterior probabilities among regions, with females having higher probability than males, but the degree of difference is negligible. The sensitivity when applying the model on the different datasets is also negligible.

\subsection{Posterior probability of in-game environmental behaviors across educational levels}

The formulas for estimating the posterior probability of in-game behaviors towards animals and plants across educational levels are as follows:

$$
\begin{aligned}
& \text { Anim } \sim \text { bernoulli(theta_anim[Edu]); } \\
& \text { Tree } \sim \text { bernoulli(theta_tree[Edu]); }
\end{aligned}
$$

Where:

- theta[1] Graduate school and higher

- theta[2] High school

- theta[3] Secondary school

- theta[4] Undergraduate school

Two formulas mentioned above can be coded and simulated using the following commands of the rstan R package (Stan Development Team, 2020):

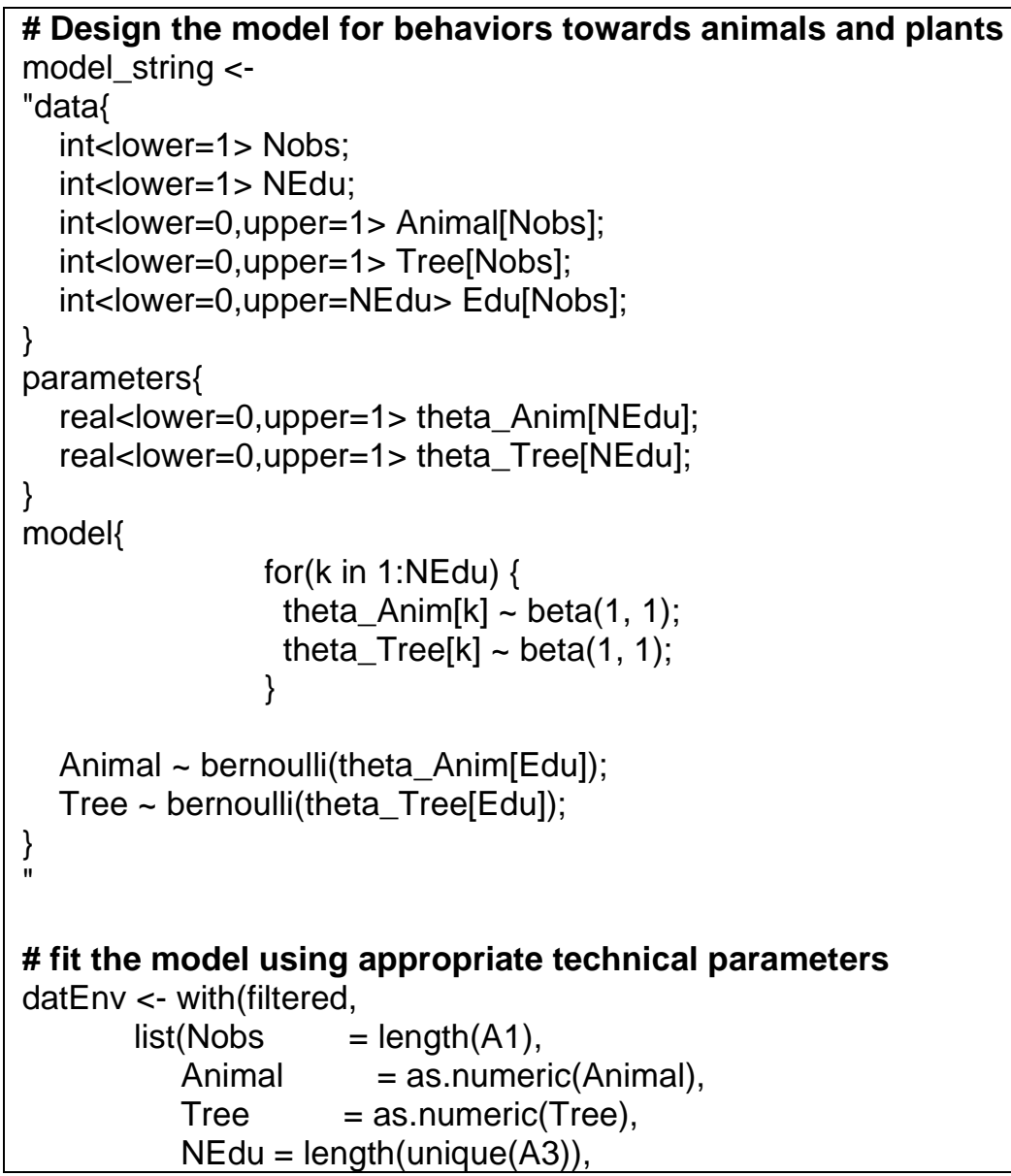

Animal bernoulli(theta_Anim[Edu]);

Tree $\sim$ bernoulli(theta_Tree[Edu]);

\}

\# fit the model using appropriate technical parameters

datEnv <- with(filtered,

$$
\begin{array}{ll}
\text { list(Nobs } & =\text { length(A1), } \\
\text { Animal } & =\text { as.numeric(Animal), } \\
\text { Tree } & =\text { as.numeric(Tree), }
\end{array}
$$$$
\mathrm{NEdu}=\text { length(unique(A3)), }
$$ 


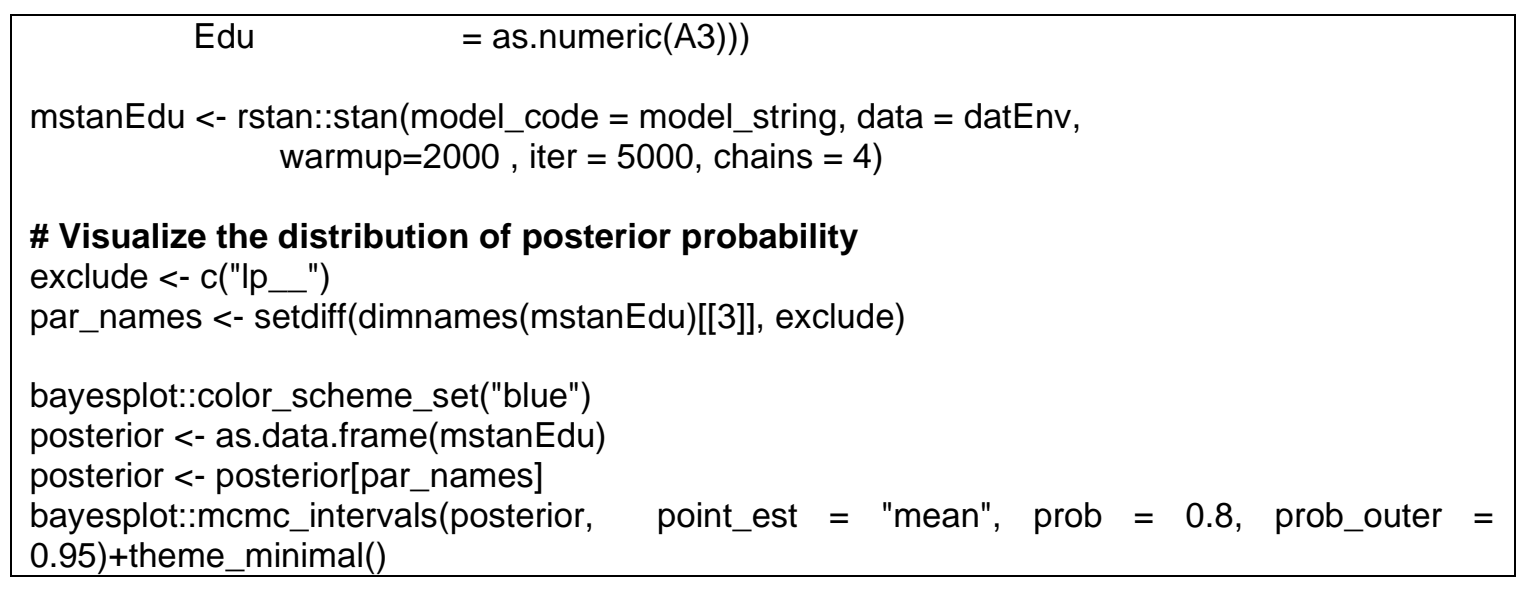

Table S3_5 presents the posterior probability of collecting all animals and plants of respondents with different educational levels employing two different datasets. The n_eff and Rhat indicate the good convergence of posteriors. The probability distribution of each educational level generated from datasets [1] and [2] is shown in Figure S3_6.

Table S3_5: Posterior probability of the in-game behavior towards animals and plants by educational levels with the prior being Beta $(1,1)$

\begin{tabular}{|c|c|c|c|c|c|c|c|c|c|c|}
\hline Dataset & Parameter & mean & sd & $\mathbf{2 . 5 \%}$ & $\mathbf{2 5 \%}$ & $\mathbf{5 0 \%}$ & $\mathbf{7 5 \%}$ & $\mathbf{9 7 . 5 \%}$ & n_eff & Rhat \\
\hline \multirow{5}{*}{ Dataset [1] } & Theta_Anim [1] & 0.97 & 0.19 & 0.92 & 0.96 & 0.97 & 0.98 & 0.99 & 20565 & 1 \\
\cline { 2 - 10 } & Theta_Anim [2] & 0.98 & 0.16 & 0.94 & 0.97 & 0.98 & 0.99 & 0.99 & 20131 & 1 \\
\cline { 2 - 10 } & Theta_Anim [3] & 0.91 & 0.09 & 0.69 & 0.87 & 0.93 & 0.97 & 0.99 & 19000 & 1 \\
\cline { 2 - 10 } & Theta_Anim [4] & 0.96 & 0.01 & 0.93 & 0.95 & 0.96 & 0.97 & 0.98 & 22965 & 1 \\
\cline { 2 - 9 } & Theta_Tree [1] & 0.97 & 0.02 & 0.92 & 0.96 & 0.97 & 0.98 & 0.99 & 21388 & 1 \\
\cline { 2 - 9 } & Theta_Tree [2] & 0.96 & 0.02 & 0.91 & 0.95 & 0.96 & 0.97 & 0.99 & 18734 & 1 \\
\cline { 2 - 9 } & Theta_Tree [3] & 0.91 & 0.09 & 0.69 & 0.87 & 0.93 & 0.97 & 1.00 & 18411 & 1 \\
\cline { 2 - 9 } & Theta_Tree [4] & 0.95 & 0.02 & 0.91 & 0.94 & 0.95 & 0.96 & 0.98 & 23012 & 1 \\
\hline \multirow{5}{*}{ Dataset [2] } & Theta_Anim [1] & 0.96 & 0.02 & 0.91 & 0.95 & 0.96 & 0.98 & 0.99 & 20269 & 1 \\
\cline { 2 - 9 } & Theta_Anim [2] & 0.98 & 0.02 & 0.93 & 0.97 & 0.98 & 0.99 & 1.00 & 19172 & 1 \\
\cline { 2 - 9 } & Theta_Anim [3] & 0.91 & 0.08 & 0.69 & 0.87 & 0.93 & 0.97 & 1.00 & 18317 & 1 \\
\cline { 2 - 9 } & Theta_Anim [4] & 0.96 & 0.02 & 0.92 & 0.95 & 0.96 & 0.97 & 0.98 & 18577 & 1 \\
\cline { 2 - 9 } & Theta_Tree [1] & 0.96 & 0.02 & 0.91 & 0.95 & 0.97 & 0.98 & 0.99 & 18273 & 1 \\
\cline { 2 - 9 } & Theta_Tree [2] & 0.95 & 0.02 & 0.90 & 0.94 & 0.96 & 0.97 & 0.99 & 19062 & 1 \\
\cline { 2 - 9 } & Theta_Tree [3] & 0.91 & 0.08 & 0.70 & 0.87 & 0.93 & 0.97 & 1.00 & 18321 & 1 \\
\cline { 2 - 9 } & Theta_Tree [4] & 0.94 & 0.02 & 0.90 & 0.93 & 0.94 & 0.96 & 0.97 & 19114 & 1 \\
\hline
\end{tabular}




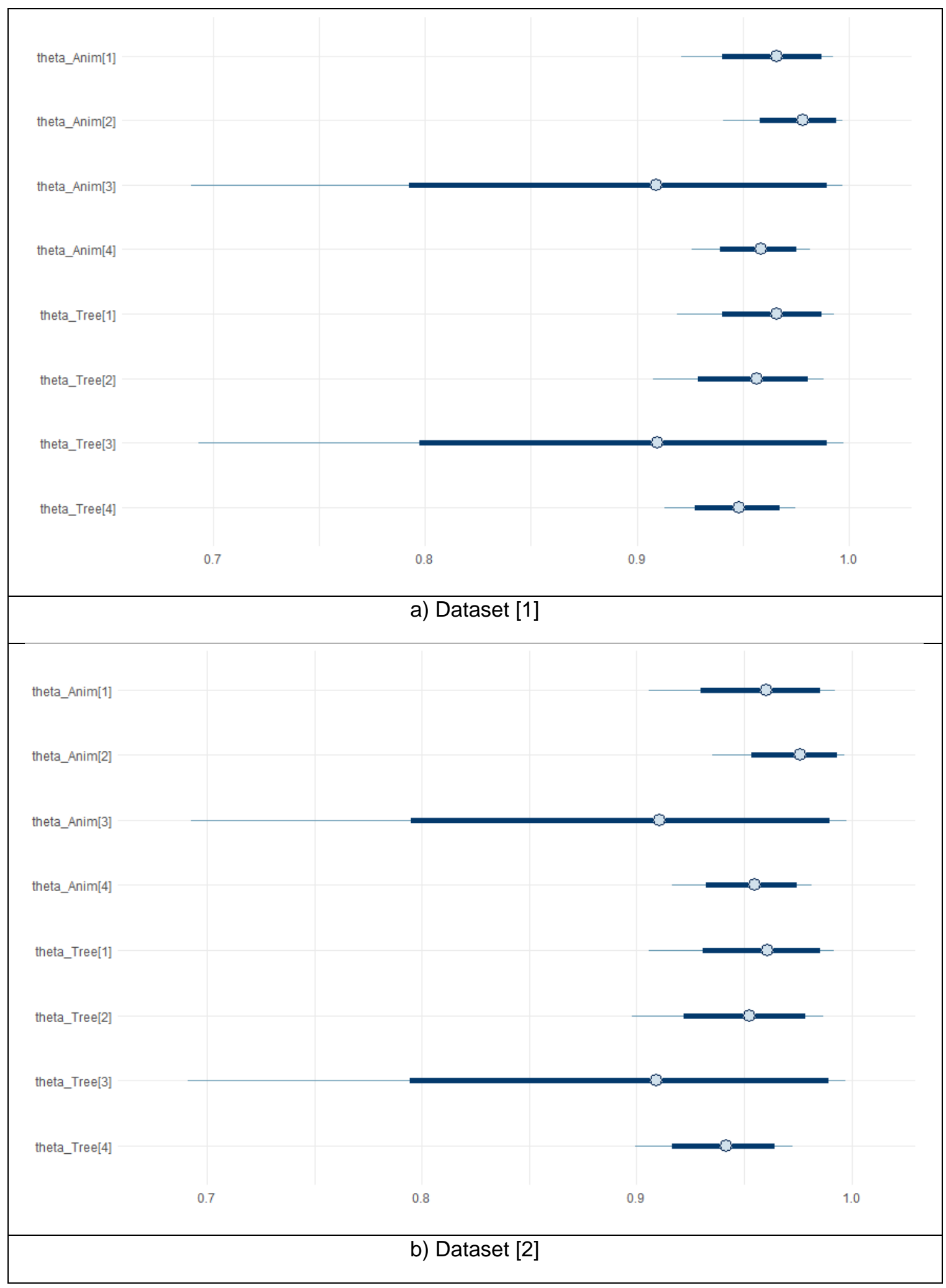

Figure S3_6: The distribution of the posterior probability of the in-game behavior towards animals and plants by educational level with the prior being Beta $(1,1)$. 
Figure S3_6a) and b) represent the distribution generated from the dataset [1] and [2], respectively. There exists the disparity of posterior probabilities among educational levels, but the degree of difference is negligible. Respondents completing high school obtain the highest likelihood, while respondents completing secondary school obtain the most varying range of likelihood. The sensitivity when applying the model on different datasets is also negligible.

\section{References}

Brooks, S. P., \& Gelman, A. (1998). General methods for monitoring convergence of iterative simulations. Journal of computational and graphical statistics, 7(4), 434455.

La, V.-P., \& Vuong, Q.-H. (2019). bayesvl: Visually learning the graphical structure of Bayesian networks and performing MCMC with 'Stan'. Retrieved from https://cran.r-project.org/web/packages/bayesvl/index.html

McElreath, R. (2020). Statistical rethinking: A Bayesian course with examples in $R$ and Stan. Boca Raton, FL, USA: CRC Press.

Stan Development Team. (2020). RStan: the R interface to Stan. Retrieved from http://mcstan.org/

Vuong, Q.-H., La, V.-P., Nguyen, M.-H., Ho, M.-T., Ho, M.-T., \& Mantello, P. (2020). Improving Bayesian statistics understanding in the age of Big Data with the bayesvl R package. Software Impacts, 100016. doi:https://doi.org/10.1016/j.simpa.2020.100016

Vuong, Q.-H., La, V.-P., Nguyen, M.-H., Ho, M.-T., Tran, T., \& Ho, M.-T. (2020). Bayesian analysis for social data: A step-by-step protocol and interpretation. MethodsX, 100924.

Vuong, Q. H. (2020). Reform retractions to make them more transparent. Nature, 582(7811), 149. doi:10.1038/d41586-020-01694-X 